\title{
Ultrasound use in the ICU for interventional pulmonology procedures
}

\author{
Ivana Milojevic ${ }^{1}$, Kewakebt Lemma ${ }^{1}$, Rahul Khosla ${ }^{2}$ \\ ${ }^{1}$ Department of Pulmonary, Critical Care and Sleep Medicine, George Washington University Medical Faculty Associates, Washington, DC, USA; \\ ${ }^{2}$ Department of Pulmonary and Critical Care Medicine, US Department of Veterans Affairs, Washington, DC, USA \\ Contributions: (I) Conception and design: All authors; (II) Administrative support: R Khosla, I Milojevic; (III) Provision of study materials or patients: \\ R Khosla; (IV) Collection and assembly of data: All authors; (V) Data analysis and interpretation: All authors; (VI) Manuscript writing: All authors; \\ (VII) Final approval of manuscript: All authors. \\ Correspondence to: Rahul Khosla, MD, MBA. Department of Pulmonary and Critical Care Medicine, US Department of Veterans Affairs, 50 Irving \\ St., NW, Room 4A-165, Washington, DC 20422, USA. Email: rahul.khosla@va.gov.
}

\begin{abstract}
Critical care ultrasound has shifted the paradigm of thoracic imaging by enabling the treating physician to acquire and interpret images essential for clinical decision-making, at the bedside, in real-time. Once considered impossible, lung ultrasound based on interpretation of artifacts along with true images, has gained momentum during the last decade, as an integral part of rapid evaluation algorithms for acute respiratory failure, shock and cardiac arrest. Procedural ultrasound image guidance is a standard of care for both common bedside procedures, and advanced procedures within interventional pulmonologist's (IP's) scope of practice. From IP's perspective, the lung, pleural, and chest wall ultrasound expertise is a prerequisite for mastery in pleural drainage techniques and transthoracic biopsies. Another ultrasound application of interest to the IP in the intensive care unit (ICU) setting is during percutaneous dilatational tracheostomy (PDT). As ICU demographics shift towards older and sicker patients, the indications for closed pleural drainage procedures, bedside transthoracic biopsies, and percutaneous dilatational tracheostomies have dramatically increased. Although ultrasound expertise is considered an essential IP operator skill there is no validated curriculum developed to address this component. Further, there is a need for developing an educational tool that matches up with the curriculum and could be integrated real-time with ultrasoundguided procedures.
\end{abstract}

Keywords: Ultrasound; interventional pulmonology (IP); intensive care unit (ICU); procedure

Submitted Jan 04, 2020. Accepted for publication May 29, 2020.

doi: $10.21037 /$ jtd-19-3564

View this article at: http://dx.doi.org/10.21037/jtd-19-3564

\section{Introduction}

The field of critical care ultrasound founded by Dr. Lichtenstein in the early 1990s evolved over three decades into an indispensable bedside tool for management of the critically ill. Out of Lichtenstein's whole-body ultrasound paradigm, lung ultrasound was born. It allowed, for the first time, rapid parenchymal assessment of this vital organ, at the point of care. Safe, non-invasive, and easily repeatable, lung ultrasound could provide immediate actionable diagnoses of life-threatening conditions, which contributed to the development of sonographic evaluation protocols for acute respiratory failure (1), shock (2-4), and cardiac arrest (5-7). Semiquantitative ultrasound assessment of lung aeration has found its application in optimization of mechanical ventilation. Ultrasound guidance is the standard of care for the majority of bedside procedures in the intensive care unit (ICU). Point-of-care ultrasound thus shifted the paradigm of thoracic imaging by eliminating the dissociation between the treating physician and physician 
interpreting the imaging (8). Although the typical role of interventional pulmonologist (IP) in the ICU is to perform advanced procedures, rather than day to day management of the critically ill, it is necessary to discuss the universal principles of lung and pleural ultrasound before focusing on sonographic aspects of pleural drainage procedures, transthoracic biopsies, and percutaneous dilatational tracheostomy (PDT).

\section{Basic principles of ultrasonography}

For a more detailed consideration of applied ultrasound physics, comprehensive review is available (9). The fundamental principle of ultrasound imaging is the reflection of ultrasound waves from tissues in the path of the beam. Echogenicity of the tissue refers to its ability to reflect and/or transmit ultrasound waves in the context of surrounding tissues. Translated to a visual greyscale, echogenic, highly reflective structures appear white, in contrast to black, non-reflective, anechoic structures; "hypoechoic", "isoechoic" and "hyperechoic" are relative descriptors of brightness of the compared structures, in shades of gray.

Whenever there is an interface of structures with different echogenic properties as defined by their acoustic impedances, a difference in brightness will be apparent on the screen. The difference in acoustic impedances between two tissues is directly proportional to the extent of reflection of ultrasound waves at their interface. Minimal acoustic impedance of air determines large impedance mismatch with surrounding tissues and results in almost complete wave reflection at lung-chest wall interface, lending the pleural line its distinctive echogenic appearance. Because only a small proportion of waves penetrates air, and air is an unfavorable medium for sound transmission, aerated lung appears as homogenous amorphic grayness rather than a discrete structural entity. As the air content in the lung decreases, acoustic mismatch also diminishes and the ultrasound beam gets partially reflected, repeatedly, as it reaches the deeper zones. With even further decrease in air content, acoustic window completely opens and lung can be directly visualized as solid parenchyma.

Pleuropulmonary pathologic processes conducive to the ultrasonographic examination such as effusions and peripherally extending consolidations provide real images, while the rest of the lung ultrasound is based on interpretation of artifacts derived by air/tissue interface.

\section{Equipment}

\section{Ultrasound machine}

Lung sonography is based on artifacts described by original investigators using technology available in the 1990s; examination can therefore be performed using any commercially available two-dimensional (2D) scanner (10-13). The lung and pleural structures are visualized in $2 \mathrm{D}$ B-mode (brightness mode, which displays the acoustic impedance of a tissue cross-section), while pleural movement can be assessed via M-mode (motion mode, which emits pulses in quick succession, taking an image each time). Image quality is determined by the individual machine characteristics, presets, and post-image processing. One caveat is that modern ultrasound machines use numerous techniques to minimize the presence of ultrasound artifacts while lung ultrasonography often relies on analysis of such artifacts. Therefore, experienced operator is expected to adapt machine settings for optimal visualization of said artifacts, unless lung presets are configured by the manufacturer. Typically, lung presets will turn off smoothing and artifact minimization algorithms such as compound and harmonic imaging, and will also reduce dynamic range. This is particularly helpful during assessment of lung sliding and B-lines. Abdominal presets may be more appropriate when evaluating pleural effusions or consolidations. Doppler ultrasound is not a part of diagnostic lung sonography paradigm, but may be useful as a procedural adjunct in order to avoid vascular injury. Preferred ultrasound devices for bedside use are easy to transport and clean, with short boot-up times and solid data storage and transfer capabilities (10).

\section{Transducers}

Ultrasound probes in use today are multiple element transducers, commonly called arrays. Various combinations of array construction (linear, convex), and array functional features such as beam scanning and steering (accomplished by electronic sequencing or electronic phasing) and beam focusing (accomplished by electronic phasing) define the probe footprint and image formats. Aside from technology used for beam manipulation which defines array type and consequently probe footprint and image format, other important probe feature is the ultrasound frequency range. Simplified terminology that partially describes probes as linear, phased array, or convex is pervasive (14) and 

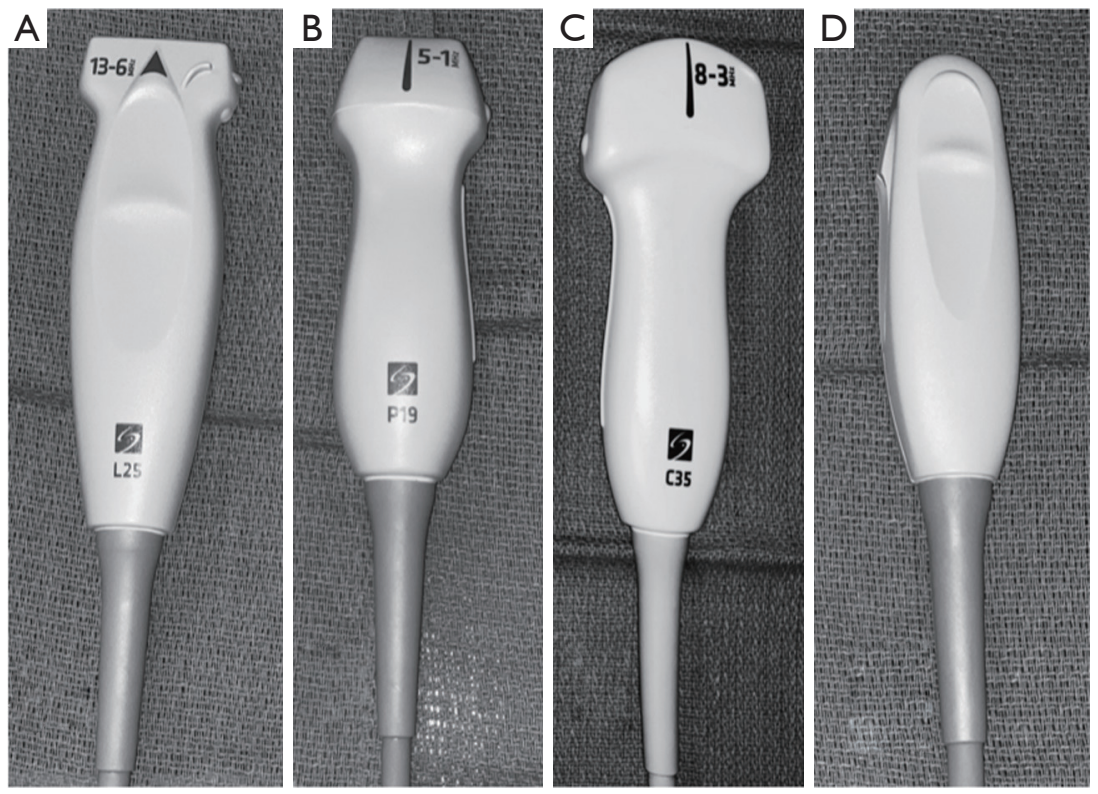

Figure 1 Various ultrasound probes. (A) Linear (vascular) probe; (B) phased array (cardiac) probe; (C) convex (abdominal) probe; (D) microconvex probe.

used interchangeably with their most prominent imaging indication — vascular, cardiac, and abdominal, respectively. Higher frequency probes are typically used to acquire high resolution images of more superficial structures, while lower frequency transducers capture lower resolution images of deeper structures. If multiple probes are available, a useful rule is to choose the one that will provide the best resolution for the required depth. Certain probes have wide frequency ranges allowing them to be more versatile and therefore cost-effective. This concept is illustrated by the whole-body critical ultrasound paradigm which was built around a universal wide range microconvex probe $(10,11)$. Intercostal acoustic windows are narrow, making it much easier to acquire images free of rib artifact using a smaller footprint probe, although diverging ultrasound beam also means that only a small field of the pleural surface is examined ("tunneled vision") and lateral resolution of the image is poor. A larger footprint enables examination of a larger field of the pleural surface and improves resolution, but it may be more difficult to acquire an image.

Linear (vascular) probes (Figure 1A) are characterized by high frequency $(7.5-10 \mathrm{MHz})$ and large footprint; they are typically used to visualize the chest wall, pleural line, and subpleural space in greater detail, and evaluate for pneumothorax. Because this evaluation is dependent on ultrasound artifacts generated by air/tissue interface, it is particularly important to use configured lung presets.

Phased-array (cardiac) probes (Figure $1 B$ ) are characterized by low frequency and small footprint allowing for relatively easy image acquisition of deeper chest structures, albeit with less image resolution and narrow field of view. Therefore, this transducer is a reasonable selection for lung ultrasound if gross and diffuse pathology is suspected.

The convex (abdominal) probes (Figure 1C) are characterized by intermediate frequency values and large footprint, allowing decent visualization of the superficial structures without losing the overview of the chest while attempting to search the entire pleural surface for localized pathology.

Microconvex probes (Figure 1D) have a small footprint in combination with good resolution for both superficial and deep structures, making them the probe of choice for lung ultrasonography $(10,11)$.

\section{Basic ultrasonography technique}

\section{Probe and image orientation}

Radiologic convention dictates that for imaging in longitudinal planes-sagittal and coronal, probe marker should be oriented towards the head (cephalad), while for 
imaging in the transverse plane probe marker should be oriented towards the patient's right. Image orientation on the screen is determined by the location of the screen marker which corresponds to the probe marker. Radiologists keep the screen marker on the left side of the screen and cardiologists traditionally keep it on the right. Critical ultrasound pioneering work was performed using head-tothe-left whole body convention including the heart, while practices among critical care practitioners are varied (15).

\section{Depth}

Prior to beginning an examination, depth should be considered. Examiner can start by visualizing the deeper structures by selecting the higher numerical depth setting and then zoom in on more superficial structures by switching to lower depth setting.

\section{Gain}

The gain function serves to optimize image quality by compensating for attenuation (sound amplitude reduction). As sound waves travel deeper into the body the amplitude of the returning signal diminishes but can be amplified by the receiver, therefore creating a brighter and better visible image on the screen. Gain can be adjusted for the near field, far field, or the entire field (overall gain). If the gain is set too low, the image appears dark, while excessive gain makes the image too bright by adding "noise".

\section{Patient positioning}

Image acquisition performed with the patient sitting up allows full access to the chest, but is rarely feasible in a critically ill patient. More often patients are imaged while supine, semi-recumbent, in lateral decubitus, or slightly rotated. Because the distribution of lung abnormalities does not rapidly change with different body positions, with the notable exception of free pleural effusions, it is possible to perform an adequate ultrasonographic exam in almost any position (13). The only true limitation is difficulty assessing the dorsal chest of a supine patient who is not easily mobilized.

\section{Scanning strategy}

By examining various ultrasound planes systematically, expert ultrasonographer can develop a 3D model of the chest with its associated pathology. Although organized approach is recommended, there is no superior scanning protocol and flexibility is allowed, to account for clinical context $(13,16)$. Universally, higher patient acuity dictates simpler and faster methodology. Comprehensive 28 rib space technique allows for semi-quantification of the interstitial syndrome $(16,17)$, while eight-zone anterolateral lung examination is often employed during qualitative evaluation for interstitial syndrome $(16,18)$. Bedside Lung Ultrasound in Emergency (BLUE) protocol assumes clinically significant interstitial syndrome and free pneumothorax should always be detected anteriorly (1), while free pleural fluid can predictably be found posteriorly above the diaphragm regardless of the volume (19). These assumptions allow rapid identification of the cause of acute respiratory failure, by examining only three identical predetermined points (the upper BLUE-point, lower BLUE-point and the posterolateral alveolar and/or pleural syndrome-or PLAPS—point) in both left and right hemithorax (20).

\section{Semiotics of lung and pleural sonography}

Basic consideration of lung and pleural sonography is that real images always reflect pathology (pleural fluid and consolidated lung parenchyma) while artifacts can reflect both physiological and pathological state.

\section{Signs}

The field of lung ultrasound is built on the following visual concepts: pleural line (bat-wing sign), lung sliding and corresponding seashore sign, absence of lung sliding and corresponding stratosphere sign, lung pulse, lung point, A-lines, B-lines, sinusoid sign, shred sign, tissue-like sign, and air bronchograms $(16,21)$. Seashore, stratosphere, and sinusoid sign are recorded in M-mode. Lung pulse and lung point can be sought for in both $\mathrm{M}$ - and B-modes. Remaining signs pertain to B-mode.

\section{Pleural line (bat-wing sign)}

The basic image of lung sonography is B-mode longitudinal view called the bat-wing sign (Figure 2) generated by upper and lower ribs (echogenic wings of the bat) and the pleural line (echogenic back of the bat). Pleural line is visualized approximately $0.5 \mathrm{~cm}$ below rib lines (Figure 2) and it always represents the parietal pleura, while visceral pleura may or may not contribute. Utilizing oblique instead of 


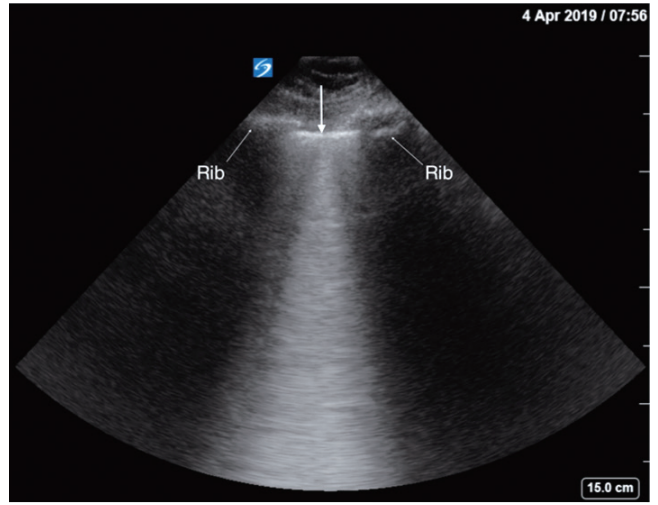

Figure 2 Bat-wing sign. Pleural line is marked by the middle arrow and corresponds to the "back of the bat". The shadows of the upper (left arrow) and lower (right arrow) ribs represent the echogenic "wings of the bat" (B-mode).

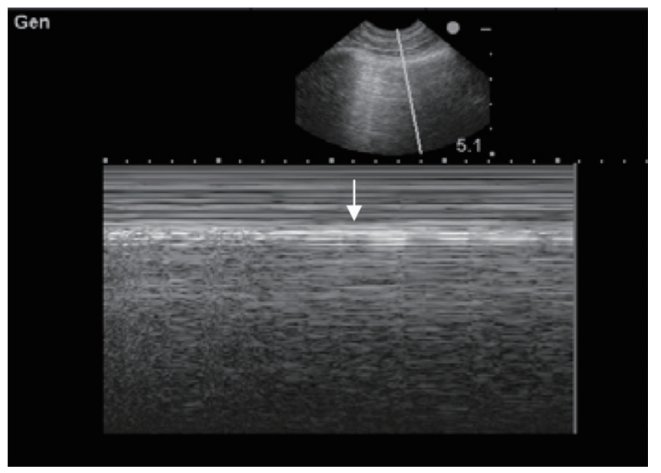

Figure 3 Seashore sign (M-mode). Below the pleural line marked by the arrow granular pattern referred to as "sandy beach" is observed and signifies that lung sliding is present.

longitudinal approach one can evaluate a larger portion of the pleural line, not interrupted by the rib shadows.

\section{Lung sliding (pleural sliding) and seashore sign}

In B-mode (Video 1), lung sliding is represented by the movement of the pleural line synchronously with respiration (13). Despite various, even contradictory definitions, lung sliding is intuitively recognized as homogeneous shimmering starting at the pleural line and spreading below (10). Characteristic appearance of lung sliding in M-mode is named seashore sign (Figure 3). Area above the pleural line with a wave-like pattern represents the chest wall and area below the pleural line with a granular/sandy beach-like pattern represents the expanded and ventilated lung. In physiologic terms, evident

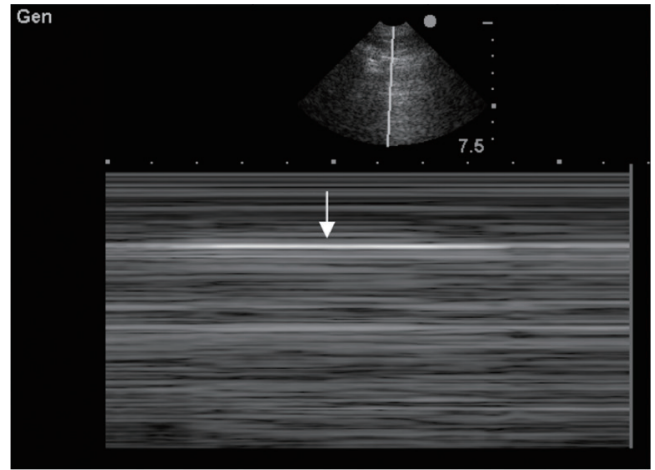

Figure 4 Barcode (stratosphere) sign (M-mode). Below the pleural line marked by the arrow, granular pattern has been replaced by horizontal lines suggesting the absence of lung sliding.

lung sliding suggests that parietal and visceral pleura are closely approximated, and regional ventilation is preserved. Presence of anterior lung sliding rules out pneumothorax with $95.3 \%$ sensitivity, $91.1 \%$ specificity and $100 \%$ negative predictive value (22). Normally, lung sliding is more subtle in the upper parts and easier to recognize towards lung bases. Certain pathologic conditions or clinical contexts can significantly reduce lung sliding; for instance, it may be quite difficult to ascertain whether lung sliding is present in a paralyzed patient, protectively ventilated with low tidal volumes (12). For optimal visualization of subtle findings during lung sliding assessment, the harmonic imaging and compound imaging filters should be disabled and dynamic range (compression) reduced, although one could consider disabling all filters (11).

\section{Absence of lung sliding (stratosphere or barcode sign)}

If lung sliding is absent, pleural line does not move synchronously with ventilation. Consequently, no shimmering is detected in B-mode (Video 2). In M-mode, the granular or sandy beach pattern below the pleural line is replaced by horizontal lines, while chest wall appearance above the pleural line is unchanged, creating the barcode or stratosphere sign (Figure 4). Detection of this sonographic pattern in the anterior chest implies that pleural surfaces are separated by air, and/or that regional ventilation is impaired by airway obstruction, hyperinflation, or pleural adhesions. Further investigation is performed by assessing for the presence of B-lines, lung point, and lung pulse (see below), while considering the clinical context. In trauma patients, for example, isolated absence of lung sliding has $98.1 \%$ sensitivity and $99.2 \%$ specificity for detection of 


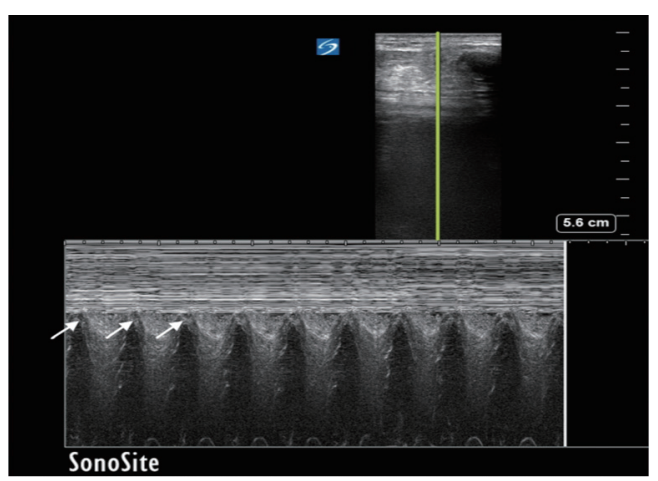

Figure 5 Lung pulse (M-mode). In the absence of lung sliding, a distinct visual pattern below the pleural line (arrows) can be seen, and is created by pleural movement synchronously with cardiac pulsations. This pattern is generated by the lack of regional ventilation and rules out pneumothorax.

pneumothorax (23). Absence of lung sliding can be observed in patients ventilated with high positive end-expiratory pressure (PEEP) but without pneumothorax (24).

\section{Lung pulse}

In the absence of lung sliding, subtle movements of the pleural line caused by the force of cardiac pulsation and synchronous with the pulse can be seen in both B-mode (Video 3) and M-mode (Figure 5). Presence of lung pulse rules out pneumothorax and confirms absence of regional ventilation with $93.0 \%$ sensitivity, and $100 \%$ specificity (25).

\section{Lung point}

Lung point marks the location on the chest wall where the collapsed lung achieves transient contact with the parietal pleura during inspiration. In B-mode, this phenomenon is appreciated as inspiratory presence and expiratory absence of lung sliding in a particular scanning location. M-mode depicts alternating seashore and stratosphere appearances in relation to the phase of the respiratory cycle (Figure 6). Sensitivity of lung point for pneumothorax detection is only $66 \%$, while specificity is $100 \%$ (26). Notably, complete lung collapse cannot produce the lung point. Location on the chest wall where lung point is detected can be used for pneumothorax semi-quantification (27).

\section{A-lines}

A-lines are hyperechoic, horizontal lines seen at regular intervals from the pleural line (Figure 7). These intervals are equal to the distance between the transducer on the

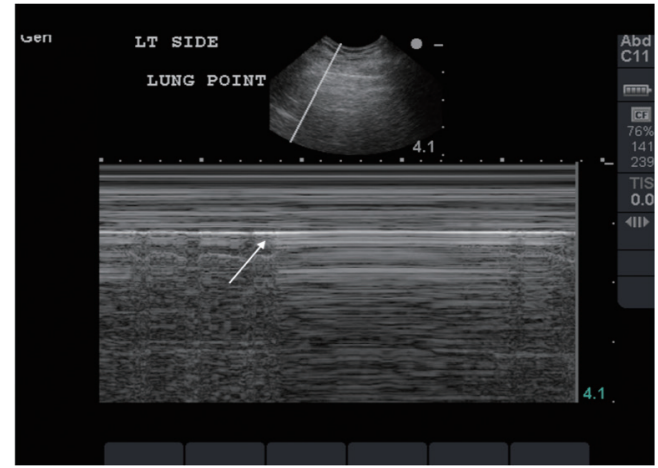

Figure 6 Lung point (M-mode). The arrow marks the transition from the seashore sign to the stratosphere sign with changes in the respiratory cycle. Seashore sign indicates that lung sliding is present during inspiration and stratosphere sign indicates that lung sliding is absent during expiration.

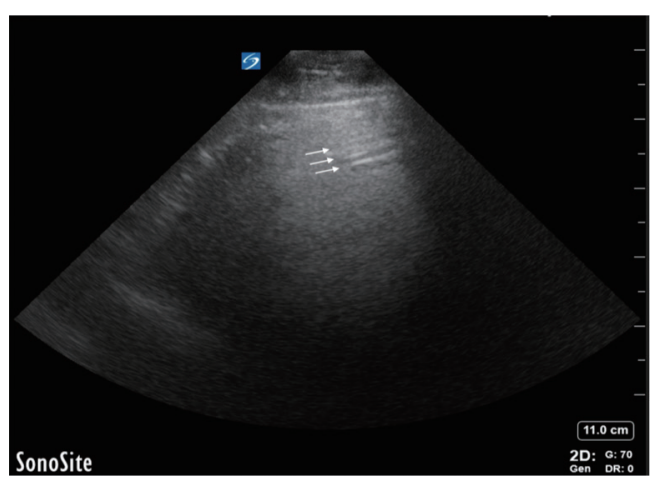

Figure 7 A-lines (B-mode). Horizontal, hyperechoic lines below the pleural line, marked by white arrows, and representing reverberation artifact.

skin and the pleural line. A-lines represent a reverberation artifact and indicate the presence of air, physiologic or free, below the parietal pleura (28). Significance of A-lines is further interpreted in the context of lung sliding and lung pulse, as well as other signs.

\section{B-lines}

B-lines (Figure 8) are discrete, laser-like, vertical, hyperechoic reverberation artifacts that arise from the pleural line, extend to the bottom of the screen without fading, erase A-lines, and move synchronously with lung sliding (16). They belong to the comet-tail artifacts family and were referred to as lung comets before the nomenclature was standardized (16). One theory of B-line genesis is that 


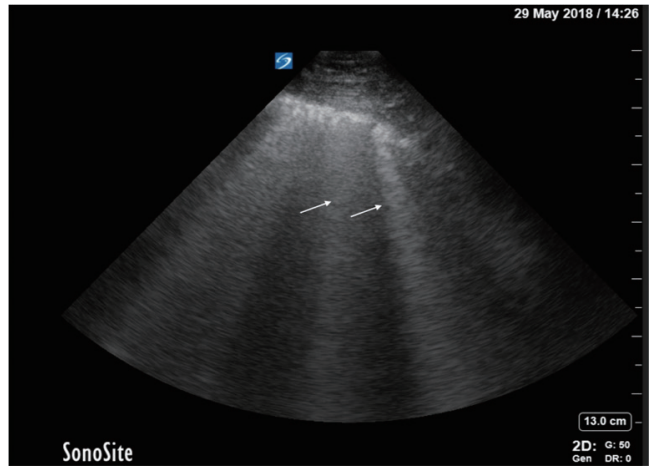

Figure 8 B-lines (B-mode). Vertical, hyperechoic lines arising from the pleural line, marked by white arrows. Visualization of more than 2 per ultrasound field is considered abnormal.

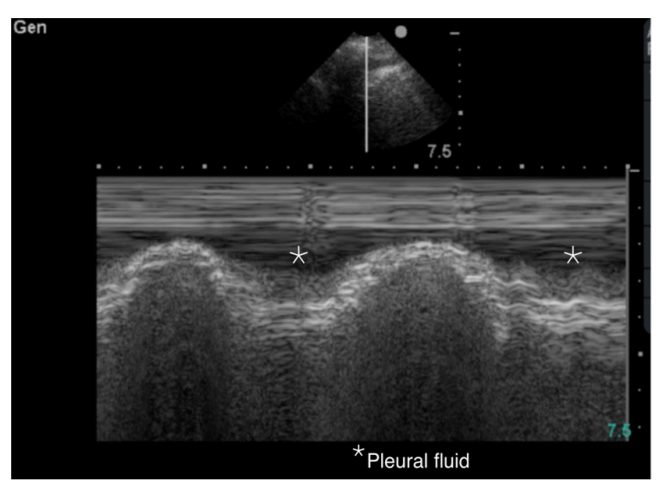

Figure 9 Sinusoidal sign. M-Mode recording of the respiratory movement of the lung and visceral pleura represented by the wavy, hyperechoic line within anechoic, free pleural fluid marked by *.

ultrasound beam interacts with edematous interlobular septa originating from the visceral pleura (29). Although anatomic and physical basis of B-lines is not certain (16), it is widely accepted that B-lines can only be seen when the visceral pleura is opposing the parietal pleura and that their presence rules out pneumothorax, even if lung sliding is absent (30). B-lines represent the densitometer of lung parenchyma, and with their increasing number lung density increases, as measured by CT (28). The very presence of B-lines is not abnormal and a maximum of $2 \mathrm{~B}$-lines per scan (longitudinal plane between two ribs) is observed at the lateral bases in $27 \%$ of healthy subjects (29). Interstitial syndrome is defined as diffuse anterolateral presence of 3 or more B-lines per scan (1). B-line distribution is another cardinal feature to consider, since focal B-pattern can be correlated with various pathologies (pneumonia, atelectasis, mass, lung contusion,

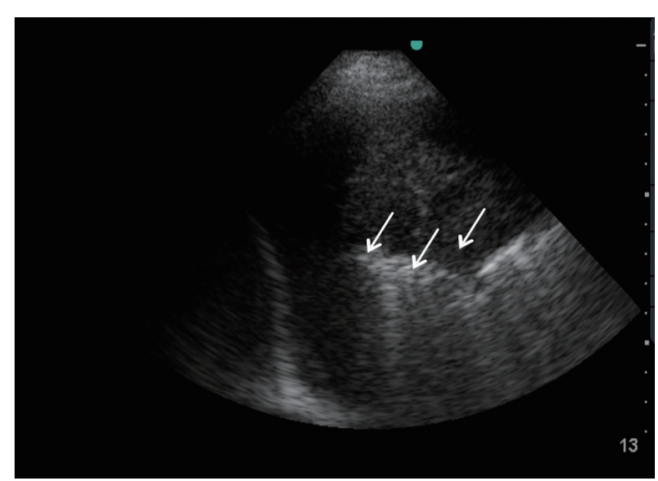

Figure 10 Shred sign (B-mode). Subpleural consolidation with echogenic and irregular borders, marked by arrows, separating it from the adjacent aerated lung parenchyma.

lung infarct) (31), while diffuse pattern of interstitial syndrome usually reflects cardiogenic pulmonary edema or ARDS.

\section{Sinusoid sign}

Sinusoid sign pertains to the M-mode recording of the lung and visceral pleura respiratory movement within free pleural fluid collection (Figure 9). Sinusoid sign also implies low viscosity of the fluid, which is important during thoracentesis deliberations (19).

\section{Shred (fractal) sign}

Shred sign is seen with smaller subpleural consolidations visualized as hypoechoic areas separated from the rest of the lung by irregular, echogenic borders (Figure 10).

These consolidations can be of infectious origin (32), due to sub-pleural infarcts (33) or diffuse lung disease (34).

\section{Tissue-like sign}

Tissue-like sign (Figure 11) represents direct visualization of the lung tissue after complete loss of aeration has occurred, typically in lobar pneumonia pattern $(35,36)$. Term sonographic hepatization of the lung effectively describes echogenicity of the consolidated lung, which is similar to that of the liver.

\section{Air-bronchogram}

Hyperechoic structure recognized within a tissue-like consolidation, which corresponds to air-filled airway (Figure 12). If air bronchogram is absent or static, potential lack of airway patency is suggested and/or partial to complete air resorption. Presence of dynamic air 
bronchograms (Video 4) supports regional airway patency and rules out atelectasis (37).

\section{Interpretation}

While sensitivity of lung ultrasound is generally robust, signs discussed above are often not specific for a diagnosis. Absent lung sliding, multiple B-lines, or lung consolidation can all be linked to various pathologic conditions and what influences interpretation the most is patient's clinical condition. Directly imaged pathologies, such as effusions and peripherally extending consolidations are usually straightforward umbrella diagnoses, while their further etiologic classification is nuanced. Artifact interpretation is typically more challenging and is based on sequential evaluations of the distribution of different signs. The final result is a sonographic lung profile, which in an appropriate clinical context is either consistent with a diagnosis, or able to rule one out. This approach is codified in several focused assessment protocols, intended for application to a correct clinical scenario and integration with standard clinical approach.

\section{Sonographic diagnoses}

\section{Normal sonographic appearance of the lung}

Presence of lung sliding in combination with A-lines and maximum of 2 B-lines per view in anterior lung fields suggests normally aerated lung parenchyma. However, the same profile can be seen with pathologies sparing the parenchyma, such as thromboembolic or airway disease, while posterior interstitial changes can be seen in the absence of true pathology, as a result of gravity alone (1).

\section{Pleural effusion}

Ultrasound can easily diagnose the effusion, suggest etiology, estimate volume, and image-guide fluid removal, making evaluation of pleural effusions the most established application of ultrasound in the chest.

With regards to pleural fluid detection, ultrasound is more accurate than clinical examination (38), since it can detect as little as $3-5 \mathrm{~mL}(39)$. Better sensitivity and reliability of ultrasound in comparison with portable chest $\mathrm{X}$-rays is of special interest in critically ill patients (40-42). Chest radiography often discriminates poorly between pleural thickening, effusion, atelectasis, pneumonia,

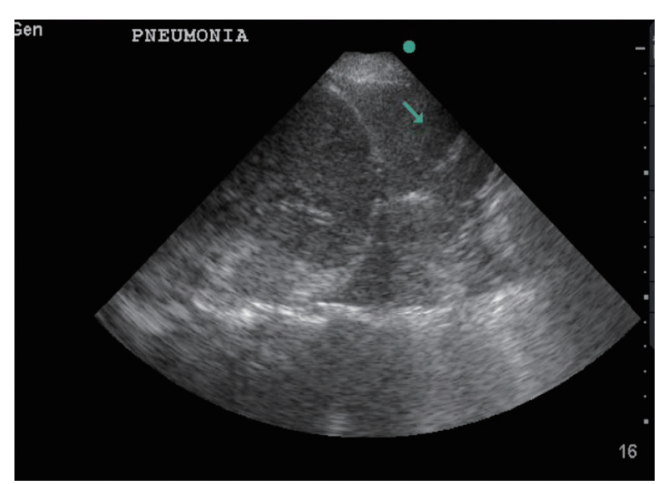

Figure 11 Tissue-like sign (B-mode). Echogenicity of the consolidated lung marked by the arrow, resembles the echogenicity of the liver, seen below the hyperechoic dome of the diaphragm.

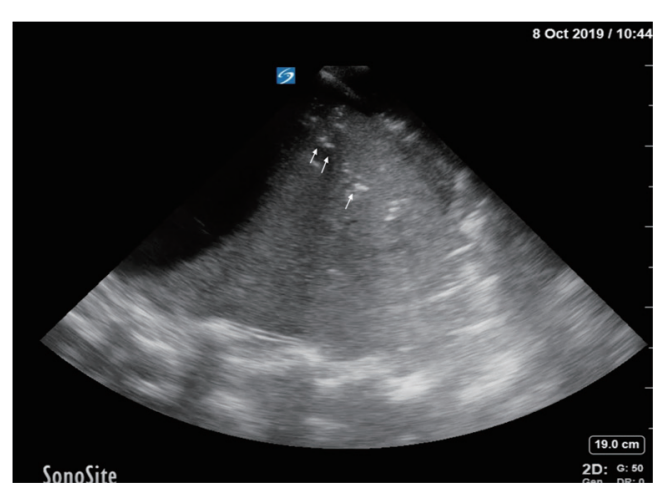

Figure 12 Air-bronchogram (B-mode). Hyperechoic flecks and lines (arrows) within consolidated lung tissue represent air filled airways.

mass, or any combination of these findings $(43,44)$, while ultrasonography is superior in differentiating pleural fluid from parenchymal processes and hemidiaphragm elevation $(39,45)$. Lung ultrasound yields diagnostic accuracy of 93\% when compared with chest CT for pleural fluid detection (46), while it outperforms CT for complex effusion diagnosis due to its superior ability to detect septations $(47,48)$.

Obeying gravity, free-flowing fluid in the pleural space accumulates in the dependent part of the chest. Posterior axillary line above the diaphragm is thus the optimal site to evaluate for a non-loculated fluid collection (16). In contrast, loculated effusions do not change with body position and can occupy non-dependent areas, warranting examination of the entire hemithorax. With probe marker oriented towards the head during longitudinal scan, 


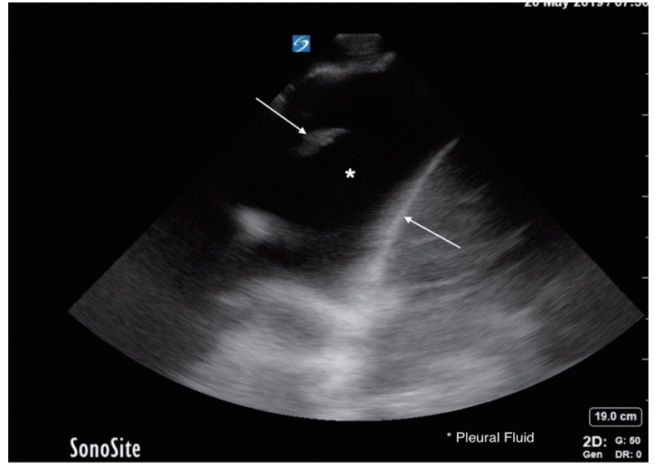

Figure 13 Pleural effusion (B-mode) seen above the diaphragm dome (right arrow) along with atelectatic lung tissue (left arrow). The anechoic pleural fluid is marked by *. This appearance is compatible with a simple effusion.

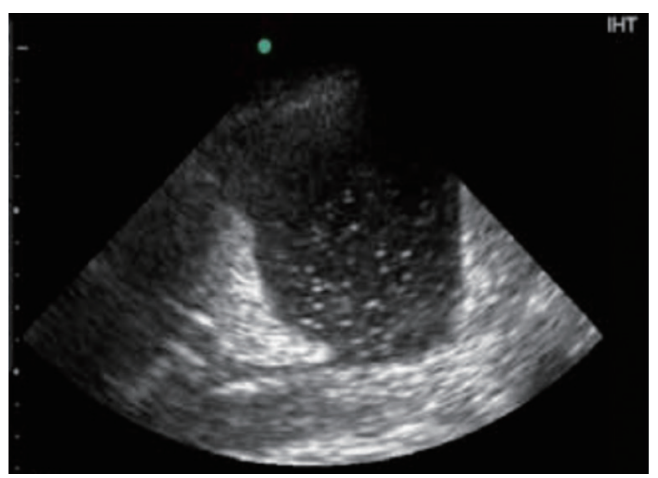

Figure 14 Complex non-septated pleural effusion (B-mode) characterized by non-homogeneous echogenic material without septations.

exam starts by identifying the diaphragm as a curvilinear, hyperechoic structure, which moves with breathing. Pleural fluid is then typically seen as an anechoic or hypoechoic collection above the diaphragm (Figure 13, Video 5). In case of a moderate to large effusion with adjacent parenchymal consolidation, tissue-like structure is noted flapping in the fluid collection (flapping lung or jellyfish sign-Video 6). Alternatively, aerated lung may be seen moving over the pleural effusion while covering liver and/or spleen during inspiration, which is called the curtain sign (Video 7). If cellular or proteinaceous debris suspended in the pleural fluid gets agitated by respiratory or cardiac motion, a swirling pattern called the plankton sign, is created. The presence of significant cellular debris is responsible for a layering effect caused by gravity, with hypocellular,

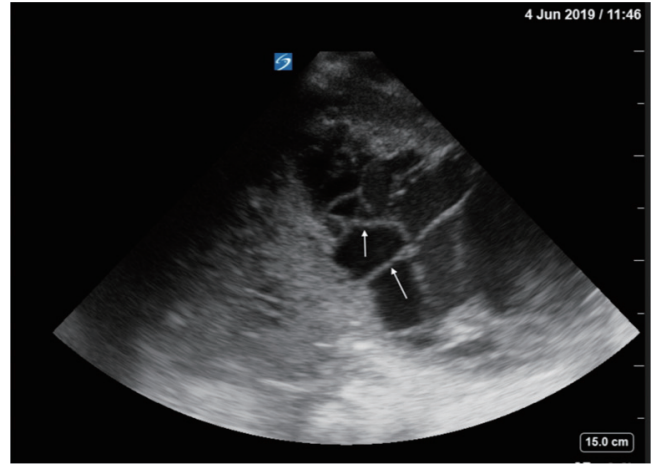

Figure 15 Complex septated pleural effusion (B-mode). Septations are indicated by the arrows.

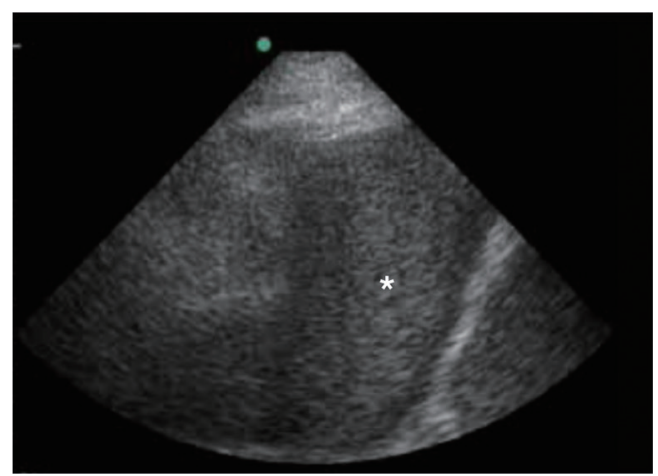

Figure 16 Homogeneous echogenic pleural effusion (B-mode). Homogeneously echogenic fluid is denoted by *.

hypoechoic top layer and cellular, hyperechoic bottom layer, which constitutes the hematocrit sign. Previously described sinusoid sign in M-mode is characteristic for a pleural effusion and can help delineate small effusions from pleural thickening, since it represents floating motion of the lung within low viscosity fluid (19).

Sonographic appearance of the pleural fluid has value in separating transudates from exudates. Four sonographic effusion patterns have been described: (I) simple, anechoic (Figure 13); (II) complex non-septated, characterized by non-homogeneous echogenic material and no septations (Figure 14); (III) complex septated, with floating fibrin strands or septae in a lattice-like pattern (Figure 15) and (IV) homogeneously echogenic (Figure 16). Transudates are almost always anechoic, while exudates can have any of the four patterns $(49,50)$. Parapneumonic effusions, empyemas, and malignant effusions can all have complex septated appearance, whereas empyemas and hemorrhagic effusions 
may appear homogeneously echogenic (51). Additional sonographic clues towards etiologic diagnosis of pleural effusion include echogenic swirling pattern and/or presence of pleural nodules which imply malignancy (52), and adjacent consolidated lung with dynamic air bronchograms which indicates infectious origin.

Despite the existence of various sonographic methods for fluid volume quantification $(41,42,53,54)$ reliable estimation of the effusion volume remains challenging for multiple reasons (55). Inspiratory interpleural distance of at least $15 \mathrm{~mm}$ with effusion also identifiable in both adjacent intercostal spaces has been suggested as a feasibility marker of safe thoracentesis in mechanically ventilated patients (19).

\section{Pneumothorax}

In mechanically ventilated or trauma patients the importance of rapid pneumothorax recognition cannot be overstated. Supine portable chest radiographs are widely available and routinely performed for this indication, albeit with a high rate of misdiagnosis (56-58). Chest CT, the gold standard for air detection in pleural space, is highly impractical for most critical care settings, not to mention the radiation exposure. The tendency of free air to collect in non-dependent anterior chest makes the supine position ideal for ultrasound exploration of pneumothorax. Bedside ultrasound is proven to be an effective tool to rule out pneumothorax $(22,25,30,59)$. Presence of anterior lung sliding virtually rules out pneumothorax in a severely dyspneic patient (22). In other words, in case of severe dyspnea/acute respiratory failure the presence of anterior lung sliding has a $100 \%$ negative predictive value for pneumothorax causing such symptoms. Absence of lung sliding, in contrast, is all but specific for pneumothorax and can also be attributed to a host of other pathologies (airway occlusion with mucous plug, tumor or foreign body, ARDS, pneumonia, effusion etc.). In the absence of lung sliding, one can still rule out pneumothorax by detecting either lung pulse (25) or B-lines (30). If not ruled out by these sequential evaluations in a critically ill patient with high pretest probability, it is safe to assume pneumothorax is present and proceed with urgent management without confirmatory testing $(16,60)$. Clinical context also weighs in heavily in post-procedural settings, where if previously present lung sliding disappears on repeated evaluation, pneumothorax is highly likely. Detection of lung point confirms the presence of pneumothorax with $100 \%$ specificity, while inability to identify lung point does not rule out pneumothorax due to insufficient sensitivity (66\%) (26). Notably, in cases of complete lung collapse lung point will not be seen, although lack of sensitivity can also be explained by suboptimal operator skills, incomplete examination, and unfavorable anatomy. Lung point location on the chest wall denotes pneumothorax extension. In one study, $90 \%$ of patients with lateral lung-points required drainage versus $8 \%$ of patients with anterior lung-point (59).

\section{Alveolar consolidation}

Alveolar consolidation extends to the pleura in overwhelming majority of cases, while $90 \%$ of consolidations may be imaged from the PLAPS point or intersection of a horizontal line at the level of the lower BLUE-point and posterior axillary line (61). Ultrasound has $90 \%$ sensitivity and $98 \%$ specificity for the diagnosis of alveolar consolidation, when compared to CT (61), and may be superior in detection of abscesses and necrotizing areas within the consolidated lung (62). Two main consolidation patterns are the smaller, non-translobar, which yields the shred sign, and the more extensive, translobar, which produces impressive image resembling anatomical lung (tissue-like sign) $(61,63)$. Since various pathological processes (compressive or obstructive atelectasis, pneumonia, ARDS, contusion, infarction or mass) can present as consolidation, additional sonographic clues may help determine the exact etiology. Different pulmonary lesions exhibit characteristic patterns of vascularization on qualitative color Doppler sonography (64). Lung pulse is reported to have a $93 \%$ sensitivity and $100 \%$ specificity for the diagnosis of complete atelectasis following right main stem intubation in patients without previous respiratory disorders (25). Identification of dynamic air bronchograms within consolidated lung indicates bronchial patency and argues against resorptive atelectasis, thus yielding $97 \%$ positive predictive value for the diagnosis of pneumonia (37). Dynamic linear-arborescent air bronchogram seems to be a specific sign for the diagnosis of ventilator-associated pneumonia $(32,65)$.

\section{Peripheral lung masses}

Malignant lesions are hypoechoic or moderately echogenic, round or oval, inhomogeneous structures with well-defined margins, often serrated or with finger-like projections into the surrounding lung tissue (Figure 17) (51). Ultrasound assessment of the chest wall invasion by the tumor has been reported to be superior to CT scan (66). Sonographic criteria 


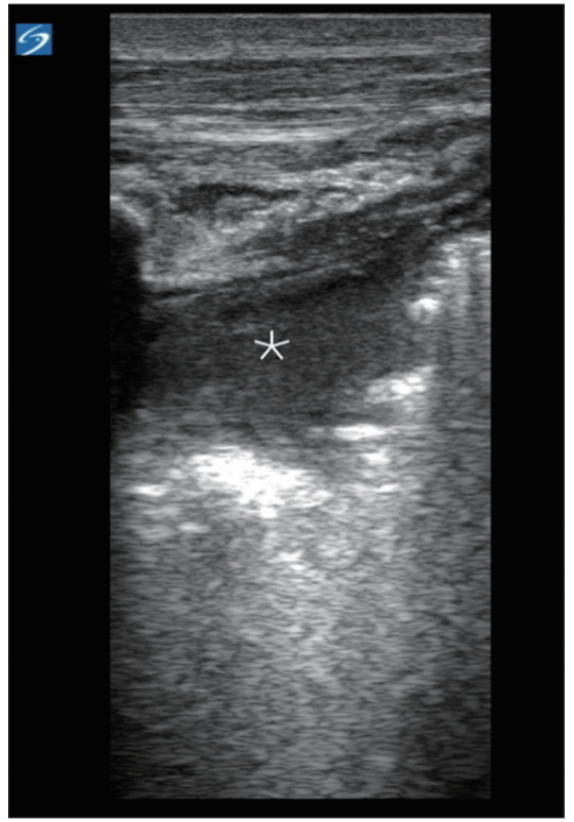

Figure 17 Peripheral lung mass (B-mode, linear probe) seen as hypoechoic lesion marked by the *, with hyperechoic, irregular margins.

for chest wall infiltration include rib destruction, pleural line interruption, and limited respiratory excursion of the mass.

\section{Alveolar-interstitial syndrome (AIS)}

The AIS is a non-specific sonographic manifestation of different acute and chronic parenchymal conditions, defined as 3 or more B-lines in a single view, diffusely disseminated over the antero-lateral thorax. In direct comparison to chest radiography, diffuse sonographic B-pattern identifies diffuse radiographic AIS with $93 \%$ sensitivity and $93 \%$ specificity (29). B-line density in a single view correlates with the severity of AIS documented by chest CT. B-lines that are $7 \mathrm{~mm}$ apart at the pleural line imply subpleural interlobular septal thickening, while B-lines that are only $3 \mathrm{~mm}$ apart at the pleural line correlate with subpleural ground glass opacities (67). Differentiation among potential causes of AIS, such as cardiogenic and noncardiogenic pulmonary edema, ARDS, interstitial pneumonia and lung fibrosis depends on additional sonographic features, but even more so on clinical context. Acute cardiogenic pulmonary edema is characterized by symmetric spatial distribution and usually progresses from the dependent zones to the anterior upper chest (13). Considering that presence of B-lines in the anterior chest denotes a severe degree of cardiogenic pulmonary edema, focused anterior scanning as suggested by the BLUE protocol is highly accurate in the critically ill, but may be insufficient to diagnose less symptomatic patients (68), which would benefit from extended examination to include the lateral chest (18). Nonhomogeneous distribution of B-lines, irregular thickened pleura with subpleural and posterior consolidations favor ARDS over cardiogenic pulmonary edema in critically ill patients (69). Semi-quantification of B-lines has found many applications in the critical care arena: cardiogenic pulmonary edema monitoring (70), assessment of hemodialysis effectiveness for extravascular lung water removal (71), fluid management in patients with ARDS and septic shock (72), predicting ARDS mortality (73), defining PEEP responders (74), identifying pronation responders $(75,76)$ and anticipating weaning failure $(77)$.

\section{BLUE protocol}

BLUE protocol is an algorithm for acute respiratory failure evaluation using bedside ultrasound which, when applied to critically ill patients yields a correct diagnosis in $90 \%$ of cases (1). Protocol combines lung and venous ultrasound in rapid, sequential assessment for lung sliding, $\mathrm{A}$ and $\mathrm{B}$-line artifacts, venous thrombosis, alveolar consolidation and/or pleural effusion, performed in 3 standardized locations per hemithorax. Combination of findings designates a profile consistent with one of the following: pulmonary edema, pulmonary embolism, pneumonia, asthma/COPD and pneumothorax.

\section{Ultrasound procedural guidance}

\section{Pleural drainage procedures}

Ultrasound reduces complications and increases the yield of thoracentesis and is integral to the management of complex pleural disease by allowing targeted placement of chest tubes. It also allows sequential sampling of fluid pockets, if $\mathrm{pH}$ variability is expected (78), while sonographic appearance informs the decision on whether to use tissue plasminogen activator with DNase therapy $(79,80)$ or recommend surgical intervention.

Rate of postprocedural pneumothorax for thoracentesis without image guidance is unacceptably high (20-39\%) and drops significantly when ultrasound is used to identify the optimal puncture site (81-83). When compared to 
clinically marked procedural site, ultrasound reduced complication rate while increasing yield, regardless of clinician seniority (38). Superiority of ultrasound-guided thoracentesis has been confirmed by meta-analysis (84) and a large retrospective cohort study (85) and adopted into practice guidelines (86). British Thoracic Society Pleural Disease Guidelines also recommend the assistance of ultrasound when placing any chest drain for fluid, although in the absence of robust data, and mostly extrapolating from thoracentesis experience.

The optimal site, angle, and depth of needle insertion portend procedure success. Lung sliding should be documented prior to needle insertion, since its absence post procedure implies iatrogenic pneumothorax. Needle trajectory must replicate the angle and position of the transducer during image acquisition, while the depth of insertion is calculated from a frozen image of the fluid pocket by using calipers. Two basic techniques are realtime guidance and site-marking technique. With realtime guidance the position of the needle is monitored constantly, allowing true targeted placement in cases of complex effusions, but is technically more challenging and not routinely performed. As long as the site marking is done correctly, and procedure performed immediately after (to avoid fluid redistribution from patient repositioning), site-marking technique has very low complication rate, even in mechanically ventilated patients (87). Intercostal arteries are not amenable to external compression and inadvertent trauma during chest drain insertion can cause bleeding into a large negative pressure space (88). The risk of injury is greatest within $6 \mathrm{~cm}$ from the spinal column $(89,90)$, justifying guideline recommendation for midaxillary placement within the "safe triangle" and along the superior rib border. This approach does not account for anatomical variation (90), the risk still being relatively high in elderly patients with tortuous arteries (91) or when obesity and subcutaneous edema render the recognition of surface landmarks difficult (92). Ability of Color Doppler to identify the intercostal vessels prior to needle insertion was reported (93) and, after testing against the gold standard CT angiogram, recommended prior to thoracentesis, when there is an increased risk of bleeding (94). This practice is not yet widely adapted, since prospective confirmation is lacking.

\section{Ultrasound-guided transthoracic needle biopsies (US-TTNB)}

US-TTNB is typically used to obtain tissue from the chest wall, parietal pleura, lung, and sometimes diaphragm or mediastinum. The technique can also be applied to the nearby extra-thoracic structures such as supraclavicular lymph nodes. US-TTNB of pleural-based lesions performed by pulmonologists is a safe procedure (95-98), with an acceptable diagnostic yield (95-97,99-101), and represents a time-saving, cost-effective alternative to CT-guided biopsy $(98,102)$. The pneumothorax rate is lower than for CTguided biopsies $(98,102)$, possibly because only peripheral lesions in direct contact with the pleura are amenable to US-TTNB. Aside from being more readily available to the critically ill patient than CT scan, a significant advantage of ultrasound is real-time guidance that allows dynamic evaluation of the target, so vasculature and necrotic areas are avoided, while biopsy of smaller lesions can be conducted in favorable respiratory phase with breath holding. The use of biopsy guide simplifies the diagnostic procedure for the operator by moving the needle in the US plane, while free hand technique should be reserved for experts (103). USTTNB has a potential role in microbiological diagnosis and/ or therapeutic drainage of a pleural-based lung abscess (104), provided there is evidence of inflammatory pleurodesis at the planned access site, so that pleural contamination is avoided. The use of ultrasound guidance during closed pleural biopsy in a challenging population of patients, including cases of failed thoracoscopy, resulted in high diagnostic accuracy (105). Although not a routine bedside procedure in the ICU setting, US-TTNB in our opinion should always be considered in critically ill patients with suspected, but, undiagnosed malignancies where a tumor mass is abutting the pleura or an enlarged lymph node is identified with US. Under such circumstances US-TTNB can be performed easily at the bedside and guide therapeutic options or help with patient prognosis.

\section{Percutaneous dilatational tracheostomy}

PDT is the procedure of choice in critically ill patients (106-108), although surgical approach may be preferred in cases of unfavorable anatomical landmarks, unstable cervical spine, malignancy at the site of insertion, and emergency situations $(108,109)$, Guidelines (108) recommend bronchoscopy use during PDT for real-time confirmation of needle entry in midline position, endotracheal tube visualization, and prevention of posterior tracheal wall injury. Reported drawbacks of bronchoscopy in this context are increase in intracranial pressure and decrease in ventilation with alveolar de-recruitment (109). Sonography can be 


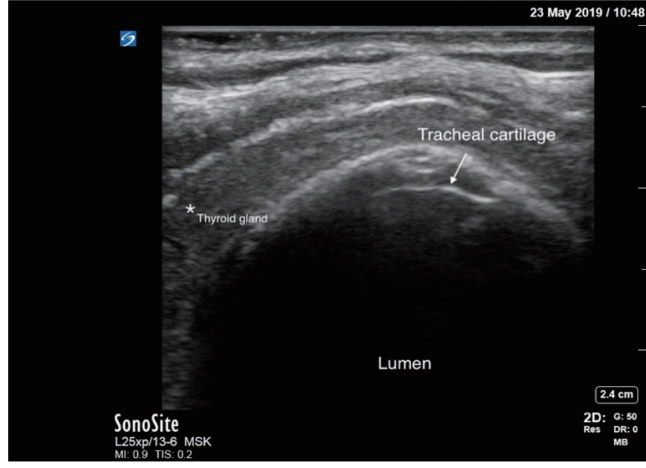

Figure 18 Trachea in the transverse plane (B-mode, linear probe).

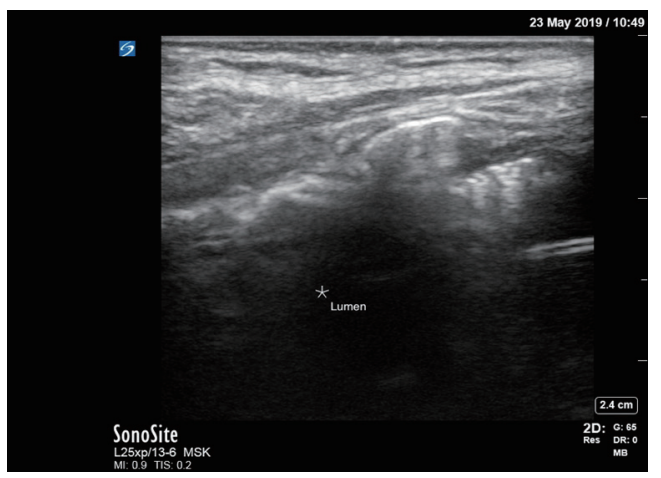

Figure 19 Trachea in the longitudinal plane with endotracheal tube in the lumen (B-mode, linear probe).

used during PDT as a neck screen, to identify patients unsuitable for the procedure and assist with landmark recognition if anatomy is difficult $(110,111)$, estimate the distance from the surface of the skin to the trachea (112), and prevent puncture of aberrant vessels. In addition, realtime ultrasound-guidance may be employed for visualization of the needle and dilator entering the trachea. Bleeding is a significant concern with PDT (113). Ultrasound allows preoperative identification of aberrant neck vessels, which in one case series resulted in changing the intended tracheal puncture site in every fourth patient (114). In our practice we routinely use US preoperatively to estimate the distance from the surface of the skin to the trachea, and to identify any aberrant vessels in the field. In real-time ultrasoundguided PDT technique, a high-frequency linear transducer is used to image proximal trachea, cricoid cartilage, cricothyroid membrane, thyroid gland, and thyroid cartilage, allowing accurate puncture of the target tracheal interspace at midline (Figures 18,19).
Ultrasound-guided PDT differs from bronchoscopicguided PDT primarily by visualization of the needle insertion, laryngoscopic endotracheal tube adjustment, and the tracheostomy tube confirmation (115). Studies comparing real-time ultrasound with bronchoscopy as PDT image guide found ultrasound guidance to be non-inferior to slightly favorable over bronchoscopy (116-118). Standalone sonographic guidance has been used in a neurosurgical population, avoiding the transient increase in intracranial pressure reported during bronchoscopy (119). However, it seemed to have an extended learning curve, with at least 50 procedures required to develop competence (120).

\section{Education and competence in chest ultrasound}

Chest sonography for both diagnostic purposes and imageguidance depends on the operator competence in image acquisition and interpretation at the point-of-care. However, there are barely any validated tools to evaluate demonstrated skills (121), no universally accepted training methodology exists, and accreditation programs are typically geared toward local clinical environments (122). Paucity of relevant literature justifies, to some extent, extrapolation from 2011 guidelines on training methodology for general critical care ultrasound (123), which were developed around American College of Chest Physicians/La Société de Reanimation de Langue Française competence statement (124). Certification programs in critical care sonography generally acknowledge the necessity of longitudinal experience needed to master ultrasound techniques (8). In 2017 Interventional Pulmonology Special Interest Group of the Thoracic Society of Australia and New Zealand developed an expert-consensus program for recognition of thoracic ultrasound competence, where competence was defined as "the ability to accurately and safely use bedside ultrasound to evaluate a pleural effusion, determine a safe site for thoracentesis or chest tube insertion, to assess pleural pathology or parenchymal lung disease and rule out a pneumothorax" (122). From IP's perspective competency requirements should be expanded to include proficiency in performing transthoracic biopsies, vascular and anterior neck imaging, with the ultimate goal being an integrated training methodology for both the use of ultrasound and the performance of ultrasound-guided procedures.

\section{Ultrasound limitations}

Since air is a poor acoustic medium, subcutaneous 
emphysema, pneumothorax and aerated lung all degrade ultrasound images and block visualization of nearby structures. Image quality may also be poor in morbidly obese or anasarcic patients. Critically ill patients tend to be immobile and can be sub-optimally positioned during exam, while the presence of catheters and dressings additionally interferes with image acquisition.

\section{Conclusions}

Past several decades have witnessed bedside ultrasonography evolve into a cornerstone of critical care medicine, while lung ultrasound gained momentum mainly in the last decade, as an integral part of rapid evaluation algorithms for acute respiratory failure, shock and cardiac arrest. Wide application of semiquantitative sonographic techniques for assessment of lung aeration in the modern ICU keeps the momentum going. Lack of radiation exposure, costeffectiveness, high diagnostic accuracy for actionable lifethreatening conditions, and point-of-care procedural image-guidance are difficult to match by other imaging technologies. In the critically ill, less invasive advanced diagnostic and therapeutic procedures involving the chest and airways are preferred over the standard surgical approach. This trend dictates increased bedside presence of the IP who must be proficient in pleural, lung, and vascular ultrasound. Ultrasound competence for the IP's scope of practice would be best enhanced by a validated training curriculum.

\section{Acknowledgments}

Funding: None.

\section{Footnote}

Provenance and Peer Review: This article was commissioned by the Guest Editors (Jonathan S. Kurman, Ashutosh Sachdeva and Rahul Nanchal) for the series "Interventional Pulmonology in the Intensive Care Unit Environment" published in Fournal of Thoracic Disease. The article has undergone external peer review.

Conflicts of Interest: All authors have completed the ICMJE uniform disclosure form (available at http://dx.doi. org/10.21037/jtd-19-3564). The series "Interventional Pulmonology in the Intensive Care Unit Environment" was commissioned by the editorial office without any funding sponsorship. The authors have no other conflicts of interest to declare.

Ethical Statement: The authors are accountable for all aspects of the work in ensuring that questions related to the accuracy or integrity of any part of the work are appropriately investigated and resolved.

Open Access Statement: This is an Open Access article distributed in accordance with the Creative Commons Attribution-NonCommercial-NoDerivs 4.0 International License (CC BY-NC-ND 4.0), which permits the noncommercial replication and distribution of the article with the strict proviso that no changes or edits are made and the original work is properly cited (including links to both the formal publication through the relevant DOI and the license). See: https://creativecommons.org/licenses/by-nc-nd/4.0/.

\section{References}

1. Lichtenstein DA, Mezière GA. Relevance of lung ultrasound in the diagnosis of acute respiratory failure: the BLUE protocol. Chest 2008;134:117-25.

2. Lichtenstein D. FALLS-protocol: lung ultrasound in hemodynamic assessment of shock. Heart Lung Vessels 2013;5:142-7.

3. Lichtenstein D. Fluid administration limited by lung sonography: the place of lung ultrasound in assessment of acute circulatory failure (the FALLS-protocol). Expert Rev Respir Med 2012;6:155-62.

4. Volpicelli G, Lamorte A, Tullio M, et al. Point-ofcare multiorgan ultrasonography for the evaluation of undifferentiated hypotension in the emergency department. Intensive Care Med 2013;39:1290-8.

5. Lichtenstein DA. How can the use of lung ultrasound in cardiac arrest make ultrasound a holistic discipline. The example of the SESAME-protocol. Med Ultrason 2014;16:252-5.

6. Lichtenstein D, Malbrain ML. Critical care ultrasound in cardiac arrest. Technological requirements for performing the SESAME-protocol--a holistic approach. Anaesthesiol Intensive Ther 2015;47:471-81.

7. Lien WC, Liu YP, Chong KM, et al. A novel US$\mathrm{CAB}$ protocol for ultrasonographic evaluation during cardiopulmonary resuscitation. Resuscitation 2017;115:e1-2.

8. Lyn-Kew KE, Koenig SJ. Bedside ultrasound for the interventional pulmonologist. Clin Chest Med 
2013;34:473-85.

9. Shriki J. Ultrasound physics. Crit Care Clin 2014;30:1-24, v.

10. Lichtenstein D. Novel approaches to ultrasonography of the lung and pleural space: where are we now? Breathe (Sheff) 2017;13:100-11.

11. Lichtenstein DA. Lung ultrasound in the critically ill. Ann Intensive Care 2014;4:1.

12. Lichtenstein DA. Lung ultrasound (in the critically ill) superior to CT: the example of lung sliding. Korean J Crit Care Med 2017;32:1-8.

13. Gargani L, Volpicelli G. How I do it: lung ultrasound. Cardiovasc Ultrasound 2014;12:25.

14. Kremkau FW. Multiple-element transducers. Radiographics 1993;13:1163-76.

15. Lichtenstein DA. Current misconceptions in lung ultrasound: a short guide for experts. Chest 2019;156:21-5.

16. Volpicelli G, Elbarbary M, Blaivas $M$, et al. International evidence-based recommendations for point-of-care lung ultrasound. Intensive Care Med 2012;38:577-91.

17. Jambrik Z, Monti S, Coppola V, et al. Usefulness of ultrasound lung comets as a nonradiologic sign of extravascular lung water. Am J Cardiol 2004;93:1265-70.

18. Volpicelli G, Mussa A, Garofalo G, et al. Bedside lung ultrasound in the assessment of alveolar-interstitial syndrome. Am J Emerg Med 2006;24:689-96.

19. Lichtenstein D, Hulot JS, Rabiller A, et al. Feasibility and safety of ultrasound-aided thoracentesis in mechanically ventilated patients. Intensive Care Med 1999;25:955-8.

20. Lichtenstein DA, Mezière GA. The BLUE-points: three standardized points used in the BLUE-protocol for ultrasound assessment of the lung in acute respiratory failure. Crit Ultrasound J 2011;3:109-10.

21. Mojoli F, Bouhemad B, Mongodi S, et al. Lung ultrasound for critically ill patients. Am J Respir Crit Care Med 2019;199:701-14.

22. Lichtenstein DA, Menu Y. A bedside ultrasound sign ruling out pneumothorax in the critically ill. Lung sliding. Chest 1995;108:1345-8.

23. Blaivas M, Lyon M, Duggal S. A prospective comparison of supine chest radiography and bedside ultrasound for the diagnosis of traumatic pneumothorax. Acad Emerg Med 2005;12:844-9.

24. Markota A, Golub J, Stožer A, et al. Absence of lung sliding is not a reliable sign of pneumothorax in patients with high positive end-expiratory pressure. Am J Emerg Med 2016;34:2034-6.

25. Lichtenstein DA, Lascols N, Prin S, et al. The "lung pulse": an early ultrasound sign of complete atelectasis.
Intensive Care Med 2003;29:2187-92.

26. Lichtenstein D, Mezière G, Biderman P, et al. The "lung point": an ultrasound sign specific to pneumothorax. Intensive Care Med 2000;26:1434-40.

27. Volpicelli G, Boero E, Sverzellati N, et al. Semiquantification of pneumothorax volume by lung ultrasound. Intensive Care Med 2014;40:1460-7.

28. Chiumello D, Mongodi S, Algieri I, et al. Assessment of lung aeration and recruitment by CT scan and ultrasound in acute respiratory distress syndrome patients. Crit Care Med 2018;46:1761-8.

29. Lichtenstein D, Mézière G, Biderman P, et al. The comettail artifact. An ultrasound sign of alveolar-interstitial syndrome. Am J Respir Crit Care Med 1997;156:1640-6.

30. Lichtenstein D, Mezière G, Biderman P, et al. The comettail artifact: an ultrasound sign ruling out pneumothorax. Intensive Care Med 1999;25:383-8.

31. Volpicelli G, Caramello V, Cardinale L, et al. Detection of sonographic B-lines in patients with normal lung or radiographic alveolar consolidation. Med Sci Monit 2008;14:CR122-8.

32. Mongodi S, Via G, Girard M, et al. Lung ultrasound for early diagnosis of ventilator-associated pneumonia. Chest 2016;149:969-80.

33. Nazerian P, Vanni S, Volpicelli G, et al. Accuracy of pointof-care multiorgan ultrasonography for the diagnosis of pulmonary embolism. Chest 2014;145:950-7.

34. Reissig A, Kroegel C. Transthoracic sonography of diffuse parenchymal lung disease: the role of comet tail artifacts. J Ultrasound Med 2003;22:173-80.

35. Cortellaro F, Colombo S, Coen D, et al. Lung ultrasound is an accurate diagnostic tool for the diagnosis of pneumonia in the emergency department. Emerg Med J 2012;29:19-23.

36. Reissig A, Copetti R, Mathis G, et al. Lung ultrasound in the diagnosis and follow-up of community-acquired pneumonia: a prospective, multicenter, diagnostic accuracy study. Chest 2012;142:965-72.

37. Lichtenstein D, Mezière G, Seitz J. The dynamic air bronchogram. A lung ultrasound sign of alveolar consolidation ruling out atelectasis. Chest 2009;135:1421-5.

38. Diacon AH, Brutsche MH, Solèr M. Accuracy of pleural puncture sites: a prospective comparison of clinical examination with ultrasound. Chest 2003;123:436-41.

39. Gryminski J, Krakówka P, Lypacewicz G. The diagnosis of pleural effusion by ultrasonic and radiologic techniques. Chest 1976;70:33-7.

40. Eibenberger KL, Dock WI, Ammann ME, et al. 
Quantification of pleural effusions: sonography versus radiography. Radiology 1994;191:681-4.

41. Roch A, Bojan M, Michelet P, et al. Usefulness of ultrasonography in predicting pleural effusions $>500$ $\mathrm{mL}$ in patients receiving mechanical ventilation. Chest 2005;127:224-32.

42. Vignon P, Chastagner C, Berkane V, et al. Quantitative assessment of pleural effusion in critically ill patients by means of ultrasonography. Crit Care Med 2005;33:1757-63.

43. Ovenfors CO, Hedgcock MW. Intensive care unit radiology. Problems of interpretation. Radiol Clin North Am 1978;16:407-39.

44. Yu CJ, Yang PC, Wu HD, et al. Ultrasound study in unilateral hemithorax opacification. Image comparison with computed tomography. Am Rev Respir Dis 1993;147:430-4.

45. Kelbel C, Börner N, Schadmand S, et al. Diagnosis of pleural effusions and atelectases: sonography and radiology compared. Rofo 1991;154:159-63.

46. Lichtenstein D, Goldstein I, Mourgeon E, et al. Comparative diagnostic performances of auscultation, chest radiography, and lung ultrasonography in acute respiratory distress syndrome. Anesthesiology 2004;100:9-15.

47. McLoud TC, Flower CD. Imaging the pleura: sonography, CT, and MR imaging. AJR Am J Roentgenol 1991;156:1145-53.

48. Kurian J, Levin TL, Han BK, et al. Comparison of ultrasound and CT in the evaluation of pneumonia complicated by parapneumonic effusion in children. AJR Am J Roentgenol 2009;193:1648-54.

49. Yang PC, Luh KT, Chang DB, et al. Value of sonography in determining the nature of pleural effusion: analysis of 320 cases. AJR Am J Roentgenol 1992;159:29-33.

50. Chen HJ, Tu CY, Ling SJ, et al. Sonographic appearances in transudative pleural effusions: not always an anechoic pattern. Ultrasound Med Biol 2008;34:362-9.

51. Khosla R. Lung Sonography. In: Thoirs K. editor. Sonography. Rijeka: IntechOpen, 2012: chapter 6. Available online: https://doi.org/10.5772/27400

52. Chian CF, Su W-L, Soh L-H, et al. Echogenic swirling pattern as a predictor of malignant pleural effusions in patients with malignancies. Chest 2004;126:129-34.

53. Balik M, Plasil P, Waldauf $\mathrm{P}$, et al. Ultrasound estimation of volume of pleural fluid in mechanically ventilated patients. Intensive Care Med 2006;32:318.

54. Remérand F, Dellamonica J, Mao Z, et al. Multiplane ultrasound approach to quantify pleural effusion at the bedside. Intensive Care Med 2010;36:656-64.
55. Brogi E, Gargani L, Bignami E, et al. Thoracic ultrasound for pleural effusion in the intensive care unit: a narrative review from diagnosis to treatment. Crit Care 2017;21:325.

56. Chiles C, Ravin CE. Radiographic recognition of pneumothorax in the intensive care unit. Crit Care Med 1986;14:677-80.

57. Tocino IM, Miller MH, Fairfax WR. Distribution of pneumothorax in the supine and semirecumbent critically ill adult. AJR Am J Roentgenol 1985;144:901-5.

58. Hill SL, Edmisten T, Holtzman G, et al. The occult pneumothorax: an increasing diagnostic entity in trauma. Am Surg 1999;65:254-8.

59. Lichtenstein DA, Mezière G, Lascols N, et al. Ultrasound diagnosis of occult pneumothorax. Crit Care Med 2005;33:1231-8.

60. Volpicelli G. Sonographic diagnosis of pneumothorax. Intensive Care Med 2011;37:224-32.

61. Lichtenstein DA, Lascols N, Mezière G, et al. Ultrasound diagnosis of alveolar consolidation in the critically ill. Intensive Care Med 2004;30:276-81.

62. Lichtenstein D, Peyrouset O. Is lung ultrasound superior to CT? The example of a CT occult necrotizing pneumonia. Intensive Care Med 2006;32:334-5.

63. Yang PC, Luh KT, Chang DB, et al. Ultrasonographic evaluation of pulmonary consolidation. Am Rev Respir Dis 1992;146:757-62.

64. Yuan A, Yang PC, Lee L, et al. Reactive pulmonary artery vasoconstriction in pulmonary consolidation evaluated by color Doppler ultrasonography. Ultrasound Med Biol 2000;26:49-56.

65. Zhou J, Song J, Gong S, et al. Lung ultrasound combined with procalcitonin for a diagnosis of ventilator-associated pneumonia. Respir Care 2019;64:519-27.

66. Bandi V, Lunn W, Ernst A, et al. Ultrasound vs. CT in detecting chest wall invasion by tumor: a prospective study. Chest 2008;133:881-6.

67. Lichtenstein DA, Mezière GA, Lagoueyte JF, et al. A-lines and B-lines: lung ultrasound as a bedside tool for predicting pulmonary artery occlusion pressure in the critically ill. Chest 2009;136:1014-20.

68. Volpicelli G, Noble VE, Liteplo A, et al. Decreased sensitivity of lung ultrasound limited to the anterior chest in emergency department diagnosis of cardiogenic pulmonary edema: a retrospective analysis. Crit Ultrasound J 2010;2:47-52.

69. Copetti R, Soldati G, Copetti P. Chest sonography: a useful tool to differentiate acute cardiogenic pulmonary edema from acute respiratory distress syndrome. 
Cardiovasc Ultrasound 2008;6:16.

70. Volpicelli G, Caramello V, Cardinale L, et al. Bedside ultrasound of the lung for the monitoring of acute decompensated heart failure. Am J Emerg Med 2008;26:585-91.

71. Noble VE, Murray AF, Capp R, et al. Ultrasound assessment for extravascular lung water in patients undergoing hemodialysis. Time course for resolution. Chest 2009;135:1433-9.

72. Caltabeloti F, Monsel A, Arbelot C, et al. Early fluid loading in acute respiratory distress syndrome with septic shock deteriorates lung aeration without impairing arterial oxygenation: a lung ultrasound observational study. Crit Care 2014;18:R91.

73. Zhao Z, Jiang L, Xi X, et al. Prognostic value of extravascular lung water assessed with lung ultrasound score by chest sonography in patients with acute respiratory distress syndrome. BMC Pulm Med 2015;15:98.

74. Constantin JM, Grasso S, Chanques G, et al. Lung morphology predicts response to recruitment maneuver in patients with acute respiratory distress syndrome. Crit Care Med 2010;38:1108-17.

75. Prat G, Guinard S, Bizien N, et al. Can lung ultrasonography predict prone positioning response in acute respiratory distress syndrome patients? J Crit Care 2016;32:36-41.

76. Wang XT, Ding X, Zhang HM, et al. Lung ultrasound can be used to predict the potential of prone positioning and assess prognosis in patients with acute respiratory distress syndrome. Crit Care 2016;20:385.

77. Soummer A, Perbet S, Brisson H, et al. Ultrasound assessment of lung aeration loss during a successful weaning trial predicts postextubation distress*. Crit Care Med 2012;40:2064-72.

78. Maskell NA, Gleeson FV, Darby M, et al. Diagnostically significant variations in pleural fluid $\mathrm{pH}$ in loculated parapneumonic effusions. Chest 2004;126:2022-4.

79. Rahman NM, Maskell NA, West A, et al. Intrapleural use of tissue plasminogen activator and DNase in pleural infection. N Engl J Med 2011;365:518-26.

80. Piccolo F, Pitman N, Bhatnagar R, et al. Intrapleural tissue plasminogen activator and deoxyribonuclease for pleural infection. An effective and safe alternative to surgery. Ann Am Thorac Soc 2014;11:1419-25.

81. Grogan DR, Irwin RS, Channick R, et al. Complications associated with thoracentesis. A prospective, randomized study comparing three different methods. Arch Intern
Med 1990;150:873-7.

82. Raptopoulos V, Davis LM, Lee G, et al. Factors affecting the development of pneumothorax associated with thoracentesis. AJR Am J Roentgenol 1991;156:917-20.

83. Barnes TW, Morgenthaler TI, Olson EJ, et al. Sonographically guided thoracentesis and rate of pneumothorax. J Clin Ultrasound 2005;33:442-6.

84. Gordon CE, Feller-Kopman D, Balk EM, et al. Pneumothorax following thoracentesis: a systematic review and meta-analysis. Arch Intern Med 2010;170:332-9.

85. Mercaldi CJ, Lanes SF. Ultrasound guidance decreases complications and improves the cost of care among patients undergoing thoracentesis and paracentesis. Chest 2013;143:532-8.

86. Havelock T, Teoh R, Laws D, et al. Pleural procedures and thoracic ultrasound: British Thoracic Society Pleural Disease Guideline 2010. Thorax 2010;65 Suppl 2:ii61-76.

87. Mayo PH, Goltz HR, Tafreshi M, et al. Safety of ultrasound-guided thoracentesis in patients receiving mechanical ventilation. Chest 2004;125:1059-62.

88. Hew M, Rahman N. Preventing intercostal vessel trauma: ultrasound to the rescue once more? Respirology 2013;18:891-2.

89. Wraight WM, Tweedie DJ, Parkin IG. Neurovascular anatomy and variation in the fourth, fifth, and sixth intercostal spaces in the mid-axillary line: a cadaveric study in respect of chest drain insertion. Clin Anat 2005;18:346-9.

90. Helm EJ, Rahman NM, Talakoub O, et al. Course and variation of the intercostal artery by CT scan. Chest 2013;143:634-9.

91. Yoneyama H, Arahata M, Temaru R, et al. Evaluation of the risk of intercostal artery laceration during thoracentesis in elderly patients by using 3D-CT angiography. Intern Med 2010;49:289-92.

92. Kanai M, Sekiguchi H. Avoiding vessel laceration in thoracentesis: a role of vascular ultrasound with color Doppler. Chest 2015;147:e5-7.

93. Salamonsen M, Ellis S, Paul E, et al. Thoracic ultrasound demonstrates variable location of the intercostal artery. Respiration 2012;83:323-9.

94. Salamonsen M, Dobeli K, McGrath D, et al. Physicianperformed ultrasound can accurately screen for a vulnerable intercostal artery prior to chest drainage procedures. Respirology 2013;18:942-7.

95. Laursen CB, Naur TM, Bodtger U, et al. Ultrasoundguided lung biopsy in the hands of respiratory physicians: diagnostic yield and complications in 215 consecutive patients in 3 centers. J Bronchology Interv Pulmonol 
2016;23:220-8.

96. García-Ortega A, Briones-Gómez A, Fabregat S, et al. Benefit of chest ultrasonography in the diagnosis of peripheral thoracic lesions in an interventional pulmonology unit. Arch Bronconeumol 2016;52:244-9.

97. Khosla R, Rohatgi PK, Seam N. Ultrasound-guided fine needle aspiration biopsy of pleural-based intrathoracic lesions. J Bronchology Interv Pulmonol 2009;16:87-90.

98. Khosla R, McLean AW, Smith JA. Ultrasound-guided versus computed tomography-scan guided biopsy of pleural-based lung lesions. Lung India 2016;33:487-92.

99. Yang PC, Chang DB, Yu CJ, et al. Ultrasound guided percutaneous cutting biopsy for the diagnosis of pulmonary consolidations of unknown aetiology. Thorax 1992;47:457-60.

100.Liao WY, Chen MZ, Chang YL, et al. US-guided transthoracic cutting biopsy for peripheral thoracic lesions less than $3 \mathrm{~cm}$ in diameter. Radiology 2000;217:685-91.

101. Khan RA, Kumar V, Taimur M, et al. Diagnostic Yield of Ultrasound-guided Trucut Biopsy in Diagnosis of Peripheral Lung Malignancies. Cureus 2019;11:e4802.

102. Sconfienza LM, Mauri G, Grossi F, et al. Pleural and peripheral lung lesions: comparison of US- and CT-guided biopsy. Radiology 2013;266:930-5.

103. Christiansen IS, Clementsen PF, Bodtger U, et al. Transthoracic ultrasound-guided biopsy in the hands of chest physicians - a stepwise approach. Eur Clin Respir J 2019;6:1579632.

104. Yang PC, Luh KT, Lee YC, et al. Lung abscesses: US examination and US-guided transthoracic aspiration. Radiology 1991;180:171-5.

105. Hallifax RJ, Corcoran JP, Ahmed A, et al. Physician-based ultrasound-guided biopsy for diagnosing pleural disease. Chest 2014;146:1001-6.

106. Kornblith LZ, Burlew CC, Moore EE, et al. One thousand bedside percutaneous tracheostomies in the surgical intensive care unit: time to change the gold standard. J Am Coll Surg 2011;212:163-70.

107. Yarmus L, Pandian V, Gilbert C, et al. Safety and efficiency of interventional pulmonologists performing percutaneous tracheostomy. Respiration 2012;84:123-7.

108. Trouillet JL, Collange O, Belafia F, et al. Tracheotomy in the intensive care unit: guidelines from a French expert panel: the French Intensive Care Society and the French Society of Anaesthesia and Intensive Care Medicine. Anaesth Crit Care Pain Med 2018;37:281-94.

109. Mehta C, Mehta Y. Percutaneous tracheostomy. Ann Card
Anaesth 2017;20:S19-25.

110. Guinot PG, Zogheib E, Petiot S, et al. Ultrasound-guided percutaneous tracheostomy in critically ill obese patients. Crit Care 2012;16:R40.

111. Muhammad JK, Patton DW, Evans RM, et al. Percutaneous dilatational tracheostomy under ultrasound guidance. Br J Oral Maxillofac Surg 1999;37:309-11.

112. Muhammad JK, Major E, Patton DW. Evaluating the neck for percutaneous dilatational tracheostomy. J Craniomaxillofac Surg 2000;28:336-42.

113. Muhammad JK, Major E, Wood A, et al. Percutaneous dilatational tracheostomy: haemorrhagic complications and the vascular anatomy of the anterior neck. A review based on 497 cases. Int J Oral Maxillofac Surg 2000;29:217-22.

114. Kollig E, Heydenreich U, Roetman B, et al. Ultrasound and bronchoscopic controlled percutaneous tracheostomy on trauma ICU. Injury 2000;31:663-8.

115.Al-Shathri Z, Susanto I. Percutaneous tracheostomy. Semin Respir Crit Care Med 2018;39:720-30.

116. Ravi PR, Vijay MN. Real time ultrasound-guided percutaneous tracheostomy: is it a better option than bronchoscopic guided percutaneous tracheostomy? Med J Armed Forces India 2015;71:158-64.

117. Gobatto ALN, Besen BAMP, Tierno PFGMM, et al. Ultrasound-guided percutaneous dilational tracheostomy versus bronchoscopy-guided percutaneous dilational tracheostomy in critically ill patients (TRACHUS): a randomized noninferiority controlled trial. Intensive Care Med 2016;42:342-51.

118.Song J, Xuan L, Wu W, et al. Comparison of percutaneous dilatational tracheostomy guided by ultrasound and bronchoscopy in critically ill obese patients. J Ultrasound Med 2018;37:1061-9.

119. Rajajee V, Fletcher JJ, Rochlen LR, et al. Real-time ultrasound-guided percutaneous dilatational tracheostomy: a feasibility study. Crit Care 2011;15:R67.

120. Petiot S, Guinot PG, Diouf M, et al. Learning curve for real-time ultrasound-guided percutaneous tracheostomy. Anaesth Crit Care Pain Med 2017;36:279-83.

121. Salamonsen M, McGrath D, Steiler G, et al. A new instrument to assess physician skill at thoracic ultrasound, including pleural effusion markup. Chest 2013;144:930-4.

122. Williamson JP, Twaddell SH, Lee YC, et al. Thoracic ultrasound recognition of competence: a position paper of the Thoracic Society of Australia and New Zealand. Respirology 2017;22:405-8.

123. Expert Round Table on Ultrasound in ICU. International 
expert statement on training standards for critical care

ultrasonography. Intensive Care Med 2011;37:1077-83.

124. Mayo PH, Beaulieu Y, Doelken P, et al. American College of Chest Physicians/La Société de Réanimation de Langue Française Statement on Competence in Critical Care Ultrasonography. Chest 2009;135:1050-60.

Cite this article as: Milojevic I, Lemma K, Khosla R. Ultrasound use in the ICU for interventional pulmonology procedures. J Thorac Dis 2021;13(8):5343-5361. doi: 10.21037/jtd19-3564 